\title{
Opportunistic Relaying for Cognitive Network with Multiple Primary Users over Nakagami-m Fading
}

\author{
Trung Q. Duong* Kyeong Jin Kim ${ }^{\dagger}$, Hans-Jürgen Zepernick*, and Chintha Tellambura ${ }^{\ddagger}$ \\ *Blekinge Institute of Technology, Karlskrona 37179, Sweden (e-mail: \{quang.trung.duong,hans-jurgen.zepernick\} @bth.se) \\ $\dagger$ Mitsubishi Electric Research Laboratories (MERL), Cambridge, MA 02139 (e-mail: kyeong.j.kim@ @otmail.com) \\ ‡University of Alberta, Edmonton AB, Canada T6G 2V4 (e-mail: chintha@ece.ualberta.ca)
}

\begin{abstract}
The performance of cognitive spectrum sharing systems with opportunistic relay selection over Nakagami- $m$ fading is analyzed in the presence of multiple primary users (PUs). In particular, we derive an exact closed-form expression for the outage probability (OP) of the considered cognitive relay systems under the joint impact of maximal transmit power $\mathcal{P}_{t}$ at secondary transmitter and peak interference power $\mathcal{I}_{p}$ at the primary user. Our general formulas cover several specific practical scenarios, e.g., where the maximal transmit power can be neglected compared to the peak interference power. In addition, a tractable expression for the asymptotic OP is also derived and reveals important insights into the system performance. We show that the number of PUs only affects the coding gain but not the diversity gain.
\end{abstract}

\section{INTRODUCTION}

The concept of relay networks has been well exploited as an efficient means to enhance the performance of wireless communications over error-prone wireless channels. Opportunistic relay selection is a breakthrough technique in wireless networks to achieve the full diversity gain in a distributed fashion with low implementation complexity [1]. As a result, the performance of opportunistic relay networks has been investigated extensively [2]-[6]. In particular, the performance of opportunistic relay selection has been derived for Rayleigh and Nakagami- $m$ fading channels in [3] and [4], respectively. It has been shown in [5] and [6] that the opportunistic amplifyand-forward (AF)/decode-and-forward (DF) relaying offers the global outage optimum for all possible relaying transmission schemes under an aggregate transmit power constraint.

Recently, cognitive radio technology has been proposed to alleviate inefficient usage of radio frequency spectrum in wireless networks [7]. In particular, under spectrum-sharing environments, the secondary user (SU) is allowed to access the radio frequency band as long as its transmit power is managed below a given threshold [8]. This value is the peak interference power constraint imposed by the primary user (PU). Extensions of opportunistic relay selection to cognitive networks have attracted great interest [9]-[11]. Specifically, the closed-form expressions of outage probability (OP), symbol error probability and ergodic capacity for opportunistic AF relaying over non identical Rayleigh fading channels have been presented in [9]. An asymptotic expression for opportunistic relaying under spectrum-sharing approach has been derived [10], which shows that the diversity gain is equal to the number of cognitive relays. Very recently, the performance of cognitive opportunistic relaying networks over frequency selective fading channels has been reported in [11]. However, all of the aforementioned works, i.e., [9]-[11], only considered Rayleigh fading channels and a single PU.

In this work, we take a step further to investigate the performance of the cognitive opportunistic relay selection scheme under spectrum-sharing environment in the presence of multiple PUs ${ }^{1}$. We derive an exact OP expression for the cognitive opportunistic relay system over Nakagami-m fading channels. Under the assumption of clustering nodes and integer fading severity parameter $m$, our analysis is valid for independent and identically distributed (i.i.d.) Nakagami$m$ fading channels. It is important to note that due to the channel gain of the link from a secondary source to the $i$ th PU, $\left|h_{\mathrm{SQ}_{i}}\right|^{2}$, the individual signal-to-noise ratio (SNR) of each relaying link contains the same random variable (RV). As such, the opportunistic relay selection for cognitive radio networks is the maximal selection of multiple statistically dependent RVs, which is cumbersome for the analysis. To overcome this difficulty, we first consider the conditional statistics on $\left|h_{\mathrm{SQ}_{i}}\right|^{2}$ and utilizing the statistical independence of the remaining RVs, which allows us to readily obtain the exact OP of the considered cognitive networks. This is the main difference in opportunistic relay selection between cognitive radio and non-cognitive radio networks. We show that when the peak interference power constraint $\mathcal{I}_{\mathrm{p}}$ imposed by the $\mathrm{PU}$ is proportional to the maximal transmit power $\mathcal{P}_{t}$ at the SU, the diversity gain of the cognitive opportunistic relaying scheme solely depends on the secondary network's parameters: i) the number of cognitive relays and ii) the fading severity of the two hops in cognitive networks. Finally, the number of PUs only determines the coding gain but not the diversity gain.

\section{System And Channel Models}

Consider a dual-hop cooperative spectrum sharing system consisting of one $\mathrm{SU}$ source $\mathrm{S}, N$ SU relays $\mathrm{R}_{k}(k=$ $1, \ldots, N)$, one SU destination $\mathrm{D}$, and $L$ PU receivers $\mathrm{Q}_{i}$, for $i \in\{1, \ldots, L\}$, as shown in Fig. 1. All terminals are singleantenna devices and operate in a half-duplex mode. In the first-hop transmission, the SU source transmits its signal $x$ to $N$ relays under a transmit power constraint which guarantees that the interference on the PU receiver $\mathrm{Q}_{i}$ does not exceed a

\footnotetext{
${ }^{1}$ The performance of cognitive relay networks with multiple primary users (PUs) has been reported in [12], [13]. However, these works only considered a single relay at the secondary network.
} 


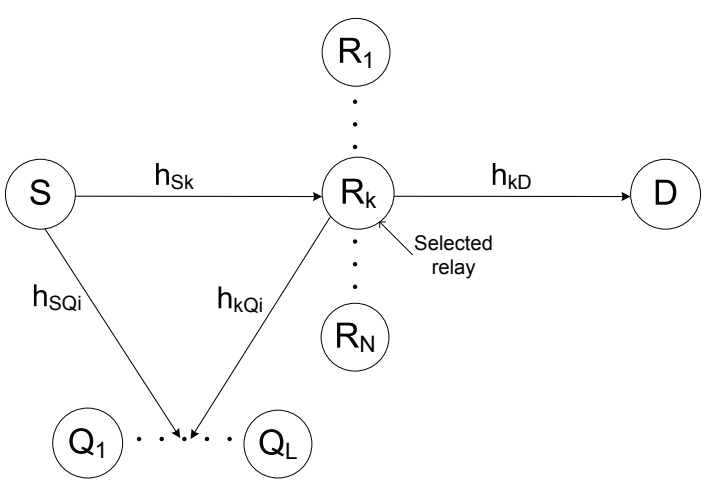

Fig. 1. Cognitive radio system with opportunistic relay selection.

threshold $\mathcal{I}_{\mathrm{p}}$. In addition, the maximal transmit power at the secondary transmitters, i.e., $\mathrm{R}_{k}$ and $\mathrm{S}$, is limited as $\mathcal{P}_{t}$. As a result, the transmit power at $\mathrm{S}$ is given by

$$
P_{\mathrm{S}}=\min \left(\mathcal{P}_{t}, \frac{\mathcal{I}_{\mathrm{p}}}{\max _{i=1, \ldots, L}\left|h_{\mathrm{SQ}_{i}}\right|^{2}}\right)
$$

where $h_{\mathrm{SQ}_{i}}$ denotes the channel coefficient of the link $\mathrm{S} \rightarrow$ $\mathrm{Q}_{i}$. In the second-hop transmission, $\mathrm{R}_{k}$ forwards the received signal from $\mathrm{S}$ to $\mathrm{D}$. Similarly, the transmit power at $\mathrm{R}_{k}$ is defined as

$$
P_{\mathrm{R}_{k}}=\min \left(\mathcal{P}_{t}, \frac{\mathcal{I}_{\mathrm{p}}}{\max _{i=1, \ldots, L}\left|h_{k \mathrm{Q}_{i}}\right|^{2}}\right)
$$

where $h_{k \mathrm{Q}_{i}}$ represents the channel coefficient of the link $\mathrm{R}_{k} \rightarrow$ $\mathrm{Q}_{i}$. Thus, the instantaneous end-to-end SNR of the link $\mathrm{S} \rightarrow$ $\mathrm{R}_{k} \rightarrow \mathrm{D}$ can be written as

$$
\gamma_{k}=\min \left(\gamma_{1 k}, \gamma_{2 k}\right)
$$

where $\gamma_{1 k}$ and $\gamma_{2 k}$ are, respectively, written as

$$
\begin{gathered}
\gamma_{1 k} \triangleq\left|h_{\mathrm{S} k}\right|^{2} \min \left(\bar{\gamma}, \frac{\bar{\gamma}_{\mathrm{p}}}{\max _{i=1, \ldots, L}\left|h_{\mathrm{SQ}_{i}}\right|^{2}}\right), \\
\gamma_{2 k} \triangleq\left|h_{k \mathrm{D}}\right|^{2} \min \left(\bar{\gamma}, \frac{\bar{\gamma}_{\mathrm{p}}}{\max _{i=1, \ldots, L}\left|h_{k \mathrm{Q}_{i}}\right|^{2}}\right),
\end{gathered}
$$

where $h_{\mathrm{S} k}$ and $h_{k \mathrm{D}}$ are the channel coefficients of the links $\mathrm{S} \rightarrow \mathrm{R}_{k}$ and $\mathrm{R}_{k} \rightarrow \mathrm{D}$, respectively. Here, the average SNR for the secondary network $\bar{\gamma}$ is given as $\bar{\gamma}=\mathcal{P}_{t} / N_{0}$ with $N_{0}$ being the noise variance; and $\bar{\gamma}_{\mathrm{p}}=\mathcal{I}_{\mathrm{p}} / N_{0}$.

The instantaneous SNR at D can be given by [2]

$$
\gamma_{\mathrm{D}}=\max _{k=1,2, \ldots, N}\left(\min \left(\gamma_{1 k}, \gamma_{2 k}\right)\right)
$$

In this paper, we assume that the fading channels experience i.i.d. Nakagami- $m$ fading. As such, the corresponding channel power gains $\left|h_{\mathrm{SQ}_{i}}\right|^{2},\left|h_{\mathrm{S} k}\right|^{2},\left|h_{k \mathrm{D}}\right|^{2}$, and $\left|h_{k \mathrm{Q}_{i}}\right|^{2}$ follow the gamma distribution, with mean powers $\Omega_{\mathrm{sq}}, \Omega_{\mathrm{sr}}, \Omega_{\mathrm{rd}}$, and $\Omega_{\mathrm{rq}}$, respectively. In other words, the cumulative distribution function (CDF) and probability density function (PDF) of RV $X$, where $X \in\left\{\left|h_{\mathrm{SQ}_{i}}\right|^{2},\left|h_{\mathrm{S} k}\right|^{2},\left|h_{k \mathrm{D}}\right|^{2},\left|h_{k \mathrm{Q}_{i}}\right|^{2}\right\}$, are given as follows:

$$
\begin{gathered}
F_{X}(x)=1-\frac{\Gamma\left(m_{X}, m_{X} x / \Omega_{X}\right)}{\Gamma\left(m_{X}\right)} \\
f_{X}(x)=\frac{x^{m_{X}-1}}{\Gamma\left(m_{X}\right)} \lambda_{X}^{m_{X}} e^{-\lambda_{X} x}
\end{gathered}
$$

where $m_{X} \in\left\{m_{\mathrm{sq}}, m_{\mathrm{sr}}, m_{\mathrm{rd}}, m_{\mathrm{rq}}\right\}$ represents the corresponding fading severity parameter $m, \lambda_{X}=\frac{m_{X}}{\Omega_{X}}$, and $\Gamma(\cdot, \cdot)$ is the incomplete gamma function [14, Eq. (8.350.2)].

\section{EXACT PERFORMANCE ANALYSIS}

For notational simplicity, let us denote the following terms $X_{1 k}=\left|h_{\mathrm{S} k}\right|^{2}, X_{2 k}=\left|h_{k \mathrm{D}}\right|^{2}, X_{3}=\max _{i=1, \ldots, L}\left|h_{\mathrm{SQ}_{i}}\right|^{2}$, and $X_{4 k}=\max _{i=1, \ldots, L}\left|h_{k Q_{i}}\right|^{2}$. Now the end-to-end SNR at D can be rewritten as

$$
\gamma_{\mathrm{D}}=\max _{k=1, \ldots, N} \min \left(\gamma_{1 k}, \gamma_{2 k}\right)
$$

where $\gamma_{1 k} \triangleq X_{1 k} \min \left(\bar{\gamma}, \frac{\bar{\gamma}_{\mathrm{p}}}{X_{3}}\right)$ and $\gamma_{2 k} \triangleq X_{2 k} \min \left(\bar{\gamma}, \frac{\bar{\gamma}_{\mathrm{p}}}{X_{4 k}}\right)$.

Corollary 1: Let $Y \in\left\{X_{3}, X_{4 k}\right\}$ be the maximum of $L$ i.i.d. gamma RVs $Y_{i}$ with parameters $\lambda_{Y}$ and $m_{Y}$, where $\lambda_{Y} \in$ $\left\{\lambda_{\mathrm{sq}}, \lambda_{\mathrm{rq}}\right\}$ and $m_{Y} \in\left\{m_{\mathrm{sq}}, m_{\mathrm{rq}}\right\}$. The CDF and PDF of the RV $Y$ are, respectively, given by [15]

$$
\begin{aligned}
& F_{Y}(y)=\widehat{\sum}_{L, u, w, m_{Y}, \lambda_{y}} y^{\tilde{l}} e^{-\lambda_{Y} u y} \\
& f_{Y}(y)=\sum_{L-1, u, w, m_{Y}, \lambda_{y}} \frac{L \lambda_{Y}^{m_{Y}}}{\Gamma\left(m_{Y}\right)} y^{m_{Y}+\widetilde{l}-1} e^{-(u+1) \lambda_{Y} y}
\end{aligned}
$$

where $\widehat{\sum_{L, u, w, m_{Y}, \lambda_{y}}}$ is a shorthand notation of

$$
\begin{aligned}
\widehat{\sum_{L, u, w, m_{Y}, \lambda_{y}}} & \triangleq \sum_{u=0}^{L}\left(\begin{array}{l}
L \\
u
\end{array}\right)(-1)^{u} \sum_{l_{1}, \ldots, l_{m_{Y}}} \frac{u !}{l_{1} !, \ldots, l_{m_{Y}} !} \\
& \times \prod_{w=0}^{m_{Y}-1}\left(\frac{\lambda_{Y}^{w}}{w !}\right)^{l_{w+1}}
\end{aligned}
$$

and $\tilde{l} \triangleq \sum_{j=0}^{m_{Y}-1} j l_{j+1}$.

It is important to note that due to the existence of $X_{3}$ in all RVs $\gamma_{k}$ for $k=1, \ldots, N, \gamma_{\mathrm{D}}$ is the maximum of $N$ dependent RVs, which is troublesome for analysis. To get around this obstacle, we first apply the conditional statistics on $X_{3}$. Thanks to the independence of the remaining RVs, i.e., $X_{1 k}, X_{2 k}$, and $X_{4 k}$, we have

$$
F_{\gamma_{\mathrm{D}}}\left(\gamma \mid X_{3}\right)=\prod_{k=1}^{N}\left[1-\left(1-F_{\gamma_{1 k}}\left(\gamma \mid X_{3}\right)\right)\left(1-F_{\gamma_{2 k}}\left(\gamma \mid X_{3}\right)\right)\right] .
$$

We first aim at deriving the conditional CDFs $F_{\gamma_{1 k}}\left(\gamma \mid X_{3}\right)$ and $F_{\gamma_{2 k}}\left(\gamma \mid X_{3}\right)$, which will be shown in the following.

Theorem 1: The conditional CDF of $\gamma_{1 k}$ is given by

$$
F_{\gamma_{1 k}}\left(\gamma \mid X_{3}\right)= \begin{cases}F_{X_{1 k}}\left(\frac{\gamma}{\bar{\gamma}}\right), & \text { for } X_{3}<\epsilon \\ F_{X_{1 k}}\left(\frac{\gamma X_{3}}{\bar{\gamma}_{\mathrm{p}}}\right), & \text { for } X_{3} \geq \epsilon\end{cases}
$$

where $\epsilon=\frac{\bar{\gamma}_{p}}{\bar{\gamma}}$. 
Proof: The proof is immediately followed from the fact that $\gamma_{1 k}=X_{1 k} \min \left(\bar{\gamma}, \frac{\bar{\gamma}_{\mathrm{p}}}{X_{3}}\right)$.

Theorem 2: The conditional CDF of $\gamma_{2 k}$ is given by

$$
F_{\gamma_{2 k}}\left(\gamma \mid X_{3}\right)=1-\Xi(\gamma)
$$

where $\Xi(\gamma)$, a function of $\gamma$ and independent of $X_{3}$, is given by

$$
\begin{aligned}
\Xi(\gamma) & =\left[1-\frac{\Gamma\left(m_{\mathrm{rq}}, \lambda_{\mathrm{rq}} \epsilon\right)}{\Gamma\left(m_{\mathrm{rq}}\right)}\right]^{L} \frac{\Gamma\left(m_{\mathrm{rd}}, \lambda_{\mathrm{rd}} \gamma / \bar{\gamma}\right)}{\Gamma\left(m_{\mathrm{rd}}\right)} \\
& +\sum_{L-1, u, l, m_{\mathrm{rq}}, \lambda_{\mathrm{rq}}} \frac{L \lambda_{\mathrm{rq}}^{m_{\mathrm{rq}}}}{\Gamma\left(m_{\mathrm{rq}}\right)} \sum_{i=0}^{m_{\mathrm{rd}}-1} \frac{1}{i !}\left(\frac{\lambda_{\mathrm{rd}} \gamma}{\bar{\gamma}_{\mathrm{p}}}\right)^{i} \\
& \times \frac{\Gamma\left(m_{\mathrm{rq}}+\widetilde{l}+i, \epsilon\left((u+1) \lambda_{\mathrm{rq}}+\frac{\lambda_{\mathrm{rd}} \gamma}{\bar{\gamma}_{\mathrm{p}}}\right)\right)}{\left[(u+1) \lambda_{\mathrm{rq}}+\frac{\lambda_{\mathrm{rd}} \gamma}{\bar{\gamma}_{\mathrm{p}}}\right]^{\left(m_{\mathrm{rq}}+\widetilde{l}+i\right)}} .
\end{aligned}
$$

Proof: The proof is given in Appendix A. Utilizing Theorem 1 and Theorem 2, we can obtain the OP $P_{\text {out }} \triangleq F_{\gamma_{\mathrm{D}}}\left(\gamma_{\text {th }}\right)$, where $\gamma_{\text {th }}$ is the outage threshold and $F_{\gamma_{\mathrm{D}}}(\cdot)$ is derived in the following theorem.

Theorem 3: The CDF of the end-to-end SNR at D is shown as (15) at the top of the next page, where $\theta \triangleq\left((u+1) \lambda_{\mathrm{sq}}+\frac{\lambda_{\mathrm{s}} k \gamma}{\bar{\gamma}_{\mathrm{p}}}\right)$.

Proof: The proof is given in Appendix B.

\section{Asymptotic Performance Analysis}

We next derive the asymptotic OP to reveal the diversity and coding gains of the considered system. In this case, we assume that the peak interference power constraint $\mathcal{I}_{\mathrm{p}}$ is proportional to the maximal transmit power $\mathcal{P}_{t}$. In other words, the ratio between these two powers, $\epsilon$, is a fixed constant.

Lemma 1: The conditional CDF of $\gamma_{1 k}$ can be asymptotically approximated as

$$
F_{\gamma_{1 k}}\left(\gamma \mid X_{3}\right) \stackrel{\bar{\gamma} \rightrightarrows \infty}{\approx}\left(\frac{\gamma}{\bar{\gamma}}\right)^{m_{\mathrm{sr}}} \begin{cases}\frac{\lambda_{\mathrm{sr}}^{m_{\mathrm{sr}}}}{\Gamma\left(m_{\mathrm{sr}}+1\right)}, & \text { for } X_{3}<\epsilon \\ \frac{\left(\lambda_{\mathrm{sr}} X_{3}\right)_{\mathrm{sr}}}{\Gamma\left(m_{\mathrm{sr}}+1\right) \epsilon^{m_{\mathrm{sr}}}}, & \text { for } X_{3} \geq \epsilon .\end{cases}
$$

Proof: We start our proof by utilizing the well-known fact that the CDF of $X$ given in (6) can be approximated as

$$
F_{X}(x) \stackrel{x \rightarrow 0}{\approx} \frac{1}{\Gamma\left(m_{X}+1\right)}\left(\frac{m_{X} x}{\Omega_{X}}\right)^{m_{X}}
$$

Then, substituting (17) into (12), we can easily conclude the proof.

Lemma 2: The conditional CDF of $\gamma_{2 k}$ can be asymptotically approximated as

$$
F_{\gamma_{2 k}}\left(\gamma \mid X_{3}\right) \stackrel{\bar{\gamma} \rightarrow \infty}{\approx} \mathcal{B}\left(\frac{\gamma}{\bar{\gamma}}\right)^{m_{\mathrm{rd}}}
$$

where $\mathcal{B} \triangleq \mathcal{B}_{1}+\mathcal{B}_{2}$, and $\mathcal{B}_{1}, \mathcal{B}_{2}$ are, respectively, shown as

$$
\begin{aligned}
& \mathcal{B}_{1} \triangleq \sum_{L-1, k, n, m_{\mathrm{rq}}, \lambda_{\mathrm{rq}}} \frac{L \lambda_{\mathrm{rd}}^{m_{\mathrm{rd}}} \lambda_{\mathrm{rq}}^{m_{\mathrm{rq}}}\left[(k+1) \lambda_{\mathrm{rq}}\right]^{-\left(m_{\mathrm{rd}}+m_{\mathrm{rq}}+\widetilde{n}\right)}}{\Gamma\left(m_{\mathrm{rd}}+1\right) \Gamma\left(m_{\mathrm{rq}}\right) \epsilon^{m_{\mathrm{rd}}}} \\
& \times \Gamma\left(m_{\mathrm{rd}}+m_{\mathrm{rq}}+\widetilde{n}, \epsilon(k+1) \lambda_{\mathrm{rq}}\right) \text { and } \\
& \mathcal{B}_{2} \triangleq\left[1-\frac{\Gamma\left(m_{\mathrm{rq}}, \lambda_{\mathrm{rq}} \epsilon\right)}{\Gamma\left(m_{\mathrm{rq}}\right)}\right]^{L} \frac{\lambda_{\mathrm{rd}}^{m_{\mathrm{rd}}}}{\Gamma\left(m_{\mathrm{rd}}+1\right) \epsilon^{m_{\mathrm{rd}}}} .
\end{aligned}
$$

Proof: The proof is given in Appendix C.

By utilizing Lemma 1 and 2, the asymptotic OP can be readily obtained through the following theorem.

Theorem 4: The asymptotic expression for the CDF of $\gamma_{D}$ is shown as (20) at the top of the next page, where $\mathcal{A}_{1} \triangleq \frac{\lambda_{\mathrm{sr}}^{m_{\mathrm{sr}}}}{\Gamma\left(m_{\mathrm{sr}}+1\right)}$, $\mathcal{A}_{2} \triangleq \frac{\lambda_{\mathrm{sr}}^{m_{\mathrm{sr}}}}{\Gamma\left(m_{\mathrm{sr}}+1\right) \epsilon^{m_{\mathrm{sr}}}}$.

Proof: The proof is given in Appendix D.

As can be observed from (20), the diversity order of opportunistic relaying for cognitive relay networks is the product between the number of cognitive relays $N$ and the minimum fading severity among the two hops. An important result inferred from the asymptotic derivation is that the number of PUs has no impact on the diversity gain, only affects the coding gain.

\section{Numerical Results AND Discussions}

In this section, numerical results are provided to illustrate the impact of primary networks on the cognitive radio systems' performance. Here, we consider the clustering networks for the cognitive relays and PUs. The outage threshold is selected as $\gamma_{\text {th }}=3 \mathrm{~dB}$. For the network topology, we consider a 2-D plane with co-linear topology for all nodes in cognitive networks. In addition, the pathloss of each channel is assumed to undergo an exponentially decaying model, where the channel mean power of the link from node $A$ to node $B, \Omega_{A B}$, is inversely proportional to their distance $d_{\mathrm{AB}}$. More specifically, $\Omega_{\mathrm{AB}} \propto d_{\mathrm{AB}}^{-\mu}$, where $\mu$ is selected as four corresponding to the free-space propagation with non line-of-sight.

Without loss of generality, we assume that the secondary source is located at the origin with coordinates $[0,0]$, whereas the secondary relays and secondary destinations are at $\left[0, \frac{1}{2}\right]$ and $[0,1]$, respectively. With this setup, we obtain $\Omega_{\mathrm{sr}}=\Omega_{\mathrm{rd}}=$ 16 under the normalization of the direct link. The channel mean power for the link to PU can be determined by $\Omega_{\mathrm{sq}}=$ $\left(\sqrt{d_{x \mathrm{q}}^{2}+d_{y \mathrm{q}}^{2}}\right)^{-4}$, where $\left[d_{x \mathrm{q}}, d_{y \mathrm{q}}\right]$ are the coordinates of the PUs. In all numerical results, the maximum interference power constraint $\mathcal{I}_{\mathrm{p}}$ is assumed to be equal to the maximum transmit power at the secondary transmitters $\mathcal{P}_{t}$, and the fading severity parameters for the link to PUs are set as $m_{\mathrm{sq}}=1$ and $m_{\mathrm{rq}}=2$. In the four figures, the analysis and asymptotic curves are plotted from (15) and (20), respectively.

Fig. 2 illustrates the impact of fading severity of the secondary networks on the outage performance. Here, both the number of relays $N$ and number of PUs $L$ are two. The fading severity parameters $m_{\mathrm{sr}}$ and $m_{\mathrm{rd}}$ are chosen according to the three cases in (20) as: i) Case 1: The first hop is less severe than the second hop $\left(m_{\mathrm{sr}}=2, m_{\mathrm{rd}}=1\right)$, ii) Case 2: The fading severity in the two hops are equal $\left(m_{\mathrm{sr}}=m_{\mathrm{rd}}=2\right)$, and iii) Case 3: The first hop is more rigorous than the second hop $\left(m_{\mathrm{sr}}=m_{\mathrm{rd}}=2\right)$. The plots in Fig. 2 confirm our result that given the fixed number of relays, the diversity order of the cognitive relay networks depends on the minimum fading severity parameter among the two hops. In comparison, the worst outage performance happens for Case 1 with the value of $\min \left(m_{\mathrm{sr}}, m_{\mathrm{rd}}\right)$ being one whereas the two Cases 2 and 3 exhibit the same diversity gain $\operatorname{since} \min \left(m_{\mathrm{sr}}, m_{\mathrm{rd}}\right)=2$. 


$$
F_{\gamma_{\mathrm{D}}}(\gamma)=\left[1-\frac{\Gamma\left(m_{\mathrm{sr}}, \lambda_{\mathrm{sr}} \gamma / \bar{\gamma}\right) \Xi(\gamma)}{\Gamma\left(m_{\mathrm{sr}}\right)}\right]^{N}\left[1-\frac{\Gamma\left(m_{\mathrm{sq}}, \lambda_{\mathrm{sq}} \epsilon\right)}{\Gamma\left(m_{\mathrm{sq}}\right)}\right]^{L}+\sum_{N, k, n, m_{\mathrm{sr}}, \lambda_{\mathrm{sr}}} \sum_{L-1, u, l, m_{\mathrm{sp}}, \lambda_{\mathrm{sq}}} \frac{L \lambda_{\mathrm{sq}}^{m_{\mathrm{sq}}} \Xi^{k}(\gamma) \Gamma\left(m_{\mathrm{sq}}+\tilde{n}+\widetilde{l}, \epsilon \theta\right) \gamma^{\tilde{n}}}{\Gamma\left(m_{\mathrm{sq}}\right) \theta^{m_{\mathrm{sq}}+\tilde{n}+\tilde{l}} \tilde{\gamma}_{\mathrm{p}}^{\tilde{n}}} .
$$

$$
\begin{aligned}
& F_{\gamma_{\mathrm{D}}}(\gamma) \stackrel{\bar{\gamma} \rightarrow \infty}{\approx}\left(\frac{\gamma}{\bar{\gamma}}\right)^{N \min \left(m_{\mathrm{sr}}, m_{\mathrm{rd}}\right)}
\end{aligned}
$$

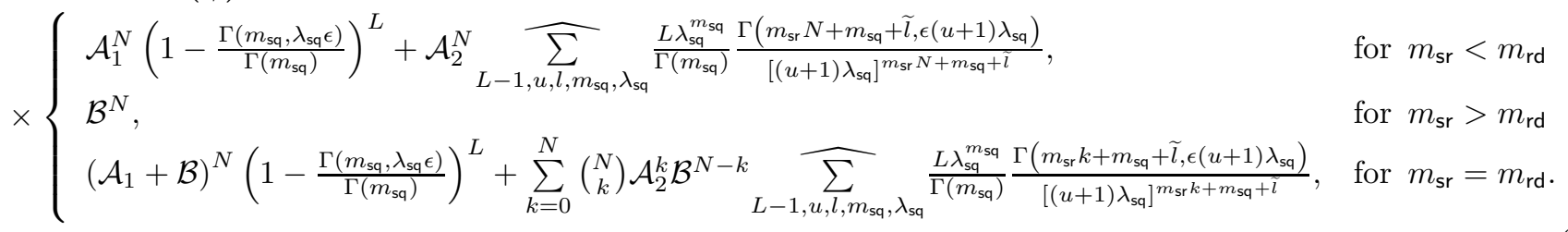

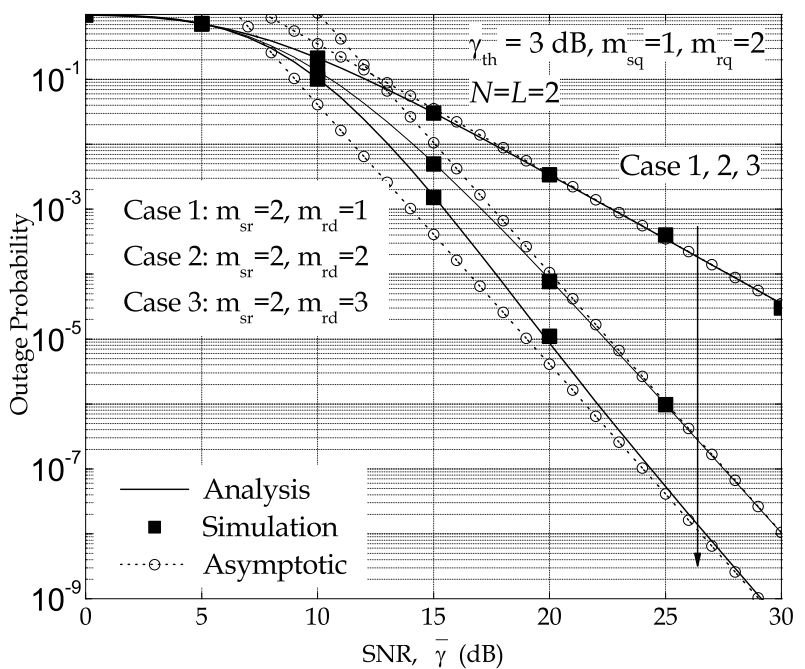

Fig. 2. OP of cognitive opportunistic relay networks in spectrum sharing condition: Varying the fading severity parameters of cognitive networks.

In Fig. 3, we investigate the effect of the number of secondary relays on the outage performance. In this scenario, the number of PUs is fixed as $L=2$, the fading severity parameters of the cognitive networks are selected as $m_{\mathrm{sr}}=3$ and $m_{\mathrm{rd}}=2$ while the number of relays $N$ is varied from one to three. As expected, the outage performance increases with the number of relays, which confirms (20).

In Fig. 4, we consider the impact of the number of PUs on the OP of the opportunistic relaying in cognitive radio networks. In this example, we fix the number of relays as $N=2$ and vary the number of PUs from one to three. Fig. 4 shows that the OP increases with the number of PUs as expected. Since as the number of PUs, $L$, increases, the transmit power constraints $P_{\mathrm{S}}$ and $P_{\mathrm{R}_{k}}$ become more and more stringent, which degrades the coding gain.

In Fig. 5, we investigate the impact of the primary network on the outage performance by varying the position of PUs from $[0.4,0.4],[0.6,0.6]$, to $[0.8,0.8]$. Intuitively, the outage of the cognitive network will decrease as the PUs move away

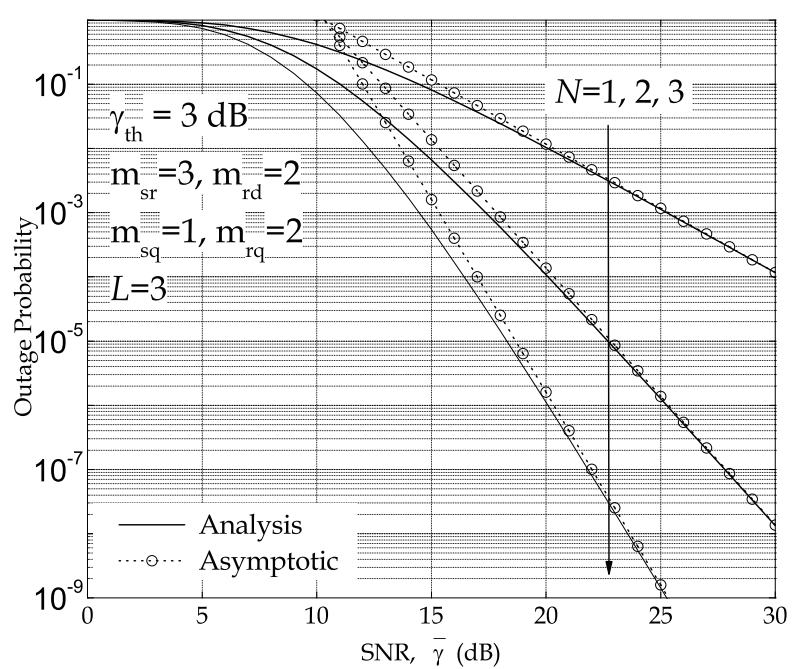

Fig. 3. OP of cognitive opportunistic relay networks in spectrum sharing condition: Varying the number of relays $N$.

from secondary nodes. Notably the three scenarios yield the same diversity order, which serves as the confirmation of the derived diversity gain, eq. (20). In addition, by comparing the cases $[0.8,0.8]$ and $[0.4,0.4]$, when the PUs move away from the cognitive network, an significant enhancement in coding gain can be achieved.

\section{Conclusions}

We have considered opportunistic relay selection for cognitive radio networks with underlay spectrum sharing and multiple PUs. Under such stringent limited interference power constraint, we derived an exact closed-form expression for the OP over Nakagami- $m$ fading channels. Utilizing this result, we can investigate the impact of primary network parameters such as the number of PUs and the positions of PUs on the cognitive network performance. In addition, to provide additional insights into the outage performance, an asymptotic $\mathrm{OP}$ as also obtained. We have concluded that the diversity gain is solely determined by the minimum fading severity among 


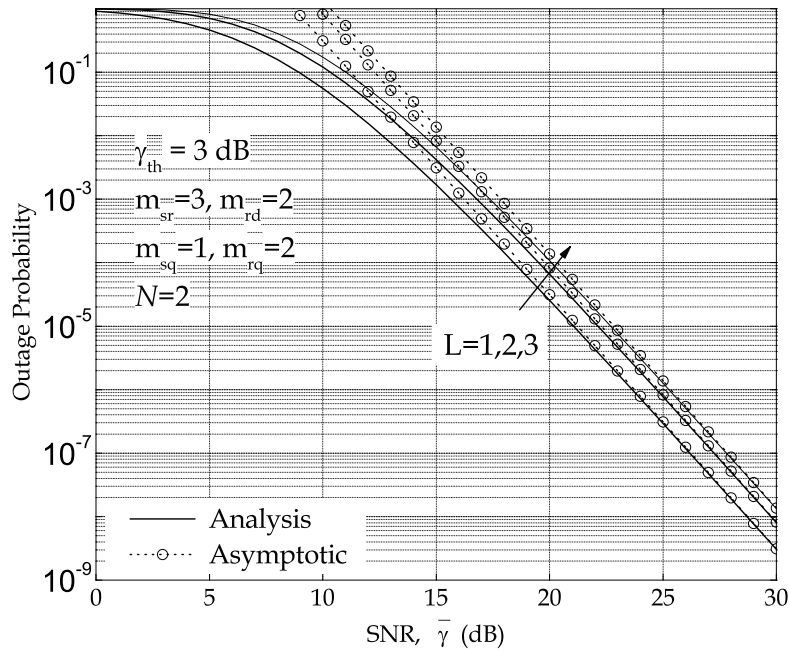

Fig. 4. OP of cognitive opportunistic relay networks in spectrum sharing condition: Varying the number of primary users $L$.

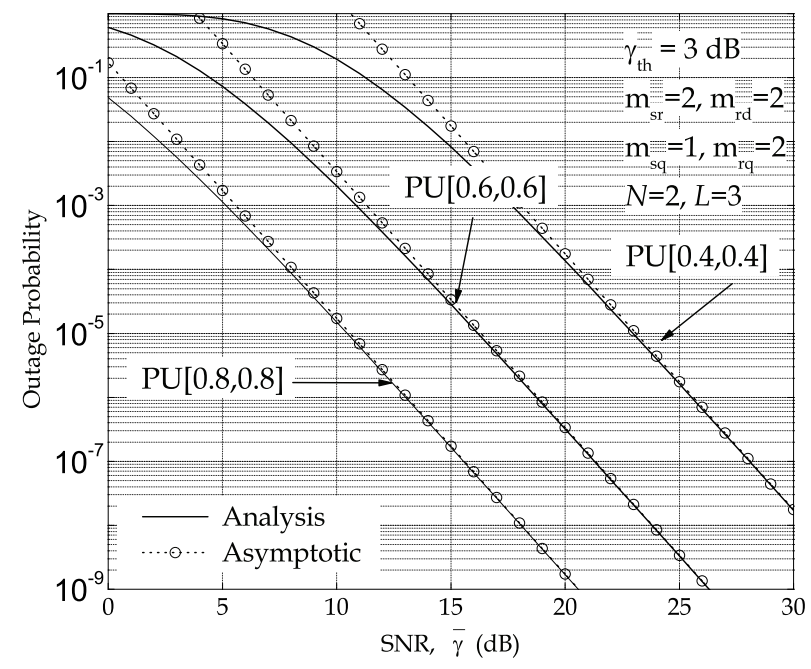

Fig. 5. OP of cognitive opportunistic relay networks in spectrum sharing condition: Varying the positions of PUs.

the two hops and the number of relays under the condition that the peak interference power is proportional to the maximal transmit power.

\section{Appendix A: Proof of Theorem 2}

Since $\gamma_{2 k}$ does not depend on $X_{3}$, we can rewrite $F_{\gamma_{2 k}}\left(\gamma \mid X_{3}\right)$ in the form of

$$
\begin{aligned}
F_{\gamma_{2 k}}\left(\gamma \mid X_{3}\right) & =F_{\gamma_{2 k}}(\gamma)=\underbrace{\operatorname{Pr}\left(\frac{X_{2 k}}{X_{4 k}}<\frac{\gamma}{\bar{\gamma}_{\mathrm{p}}}, X_{4 k}>\epsilon\right)}_{\mathcal{I}_{1}} \\
& +\underbrace{\operatorname{Pr}\left(X_{2 k}<\frac{\gamma}{\bar{\gamma}}, X_{4 k} \leq \epsilon\right)}_{\mathcal{I}_{2}} .
\end{aligned}
$$

The first summand in (A.1) can be calculated as

$$
\mathcal{I}_{1}=\int_{\epsilon}^{\infty} f_{X_{4 k}}\left(x_{4}\right) \int_{0}^{\frac{\gamma x_{4}}{\bar{\gamma}_{\mathrm{p}}}} f_{X_{2 k}}\left(x_{2}\right) d x_{2} d x_{4} \text {. }
$$

By substituting (6) into (A.2), we obtain

$$
\mathcal{I}_{1}=1-F_{X_{4 k}}(\epsilon)-\int_{\epsilon}^{\infty} \frac{\Gamma\left(m_{\mathrm{rd}}, \frac{\lambda_{\mathrm{rd}} \gamma x_{4}}{\bar{\gamma}_{\mathrm{p}}}\right)}{\Gamma\left(m_{\mathrm{rd}}\right)} f_{X_{4 k}}\left(x_{4}\right) d x_{4} .
$$

Using [14, Eq. (8.352.2)] to expand the incomplete Gamma function in terms of a finite sum and substituting (10) into (A.3), we get

$$
\begin{aligned}
& \mathcal{I}_{1}=1-F_{X_{4 k}}(\epsilon)-\sum_{L-1, u, l, m_{\mathrm{rq}}, \lambda_{\mathrm{rq}}} \frac{L \lambda_{\mathrm{rq}}^{m_{\mathrm{rq}}}}{\Gamma\left(m_{\mathrm{rq}}\right)} \sum_{i=0}^{m_{\mathrm{rd}}-1} \frac{1}{i !}\left(\frac{\lambda_{\mathrm{rd}} \gamma}{\bar{\gamma}_{\mathrm{p}}}\right)^{i} \\
& \times \frac{\Gamma\left(m_{\mathrm{rq}}+\tilde{l}+i, \epsilon\left((u+1) \lambda_{\mathrm{rq}}+\frac{\lambda_{\mathrm{rd}} \gamma}{\bar{\gamma}_{\mathrm{p}}}\right)\right)}{\left[(u+1) \lambda_{\mathrm{rq}}+\frac{\lambda_{\mathrm{rd}} \gamma}{\bar{\gamma}_{\mathrm{p}}}\right]^{\left(m_{\mathrm{rq}}+\tilde{l}+i\right)}}
\end{aligned}
$$

after some manipulations. In addition, it is easy to see that

$$
\mathcal{I}_{2}=F_{X_{4 k}}(\epsilon) F_{X_{2 k}}\left(\frac{\gamma}{\bar{\gamma}}\right) \text {. }
$$

By pulling (A.1), (A.4), and (A.5) together, yields (13), which finalizes the proof.

\section{APPENDIX B: PRoOf OF THEOREM 3}

The CDF of $\gamma_{D}$ can be given by

$$
\begin{aligned}
F_{\gamma_{\mathrm{D}}}(\gamma) & =\underbrace{\int_{0}^{\epsilon} F_{\gamma_{\mathrm{D}}}\left(\gamma \mid X_{3}\right) f_{X_{3}}\left(x_{3}\right) d x_{3}}_{\mathcal{J}_{1}} \\
& +\underbrace{\int_{\epsilon}^{\infty} F_{\gamma_{\mathrm{D}}}\left(\gamma \mid X_{3}\right) f_{X_{3}}\left(x_{3}\right) d x_{3}}_{\mathcal{J}_{2}}
\end{aligned}
$$

where $F_{\gamma_{\mathrm{D}}}\left(\gamma \mid X_{3}\right)$ is readily expressed as

$$
F_{\gamma_{\mathrm{D}}}\left(\gamma \mid X_{3}\right)=\left[1-\left(1-F_{\gamma_{1 k}}\left(\gamma \mid X_{3}\right)\right)\left(1-F_{\gamma_{2 k}}\left(\gamma \mid X_{3}\right)\right)\right]^{N} \text {. }
$$

To compute the two above integrals in (B.1), we make use of Theorems 1 and 2. For $\mathcal{J}_{1}$, it is observed from (12) that $F_{\gamma_{1 k}}\left(\gamma \mid X_{3}\right)$ is independent of $X_{3}$, which leads to

$$
\begin{aligned}
& \mathcal{J}_{1}=\int_{0}^{\epsilon}\left[1-\frac{\Gamma\left(m_{\mathrm{sr}}, \lambda_{\mathrm{sr}} \gamma / \bar{\gamma}\right)}{\Gamma\left(m_{\mathrm{sr}}\right)} \Xi(\gamma)\right]^{N} f_{X_{3}}\left(x_{3}\right) d x_{3} \\
& =\left[1-\frac{\Gamma\left(m_{\mathrm{sr}}, \lambda_{\mathrm{sr}} \gamma / \bar{\gamma}\right)}{\Gamma\left(m_{\mathrm{sr}}\right)} \Xi(\gamma)\right]^{N}\left[1-\frac{\Gamma\left(m_{\mathrm{sq}}, \lambda_{\mathrm{sq}} \epsilon\right)}{m_{\mathrm{sq}}}\right]^{L} .
\end{aligned}
$$

Next, to computer $\mathcal{J}_{2}$, we substitute (12) into (B.2), which results in

$$
\mathcal{J}_{2}=\int_{\epsilon}^{\infty}\left[1-\frac{\Gamma\left(m_{\mathrm{sr}}, \lambda_{\mathrm{sr}} \gamma x_{3} / \bar{\gamma}_{\mathrm{p}}\right)}{\Gamma\left(m_{\mathrm{sr}}\right)} \Xi(\gamma)\right]^{N} f_{X_{3}}\left(x_{3}\right) d x_{3} .
$$

Then, plugging (10) into (B.5) and using [14, Eq. (8.352.2)] together with the multinomial expansion, $\mathcal{J}_{2}$ is derived as

$$
\begin{aligned}
& \mathcal{J}_{2}=\sum_{N, k, n, m_{\mathrm{sr}}, \lambda_{\mathrm{sr}}} \sum_{L-1, u, l, m_{\mathrm{sq}}, \lambda_{\mathrm{sq}}} \frac{L \lambda_{\mathrm{sq}}^{m_{\mathrm{sq}}} \Xi^{k}(\gamma)}{\Gamma\left(m_{\mathrm{sq}}\right)}\left(\frac{\gamma}{\bar{\gamma}_{\mathrm{p}}}\right)^{\tilde{n}} \\
& \times \int_{\epsilon}^{\infty} x_{3}^{m_{\mathrm{sq}}+\widetilde{n}+\tilde{l}-1} \exp \left[-\left((u+1) \lambda_{\mathrm{sq}}+\frac{\lambda_{\mathrm{sr}} k \gamma}{\bar{\gamma}_{\mathrm{p}}}\right) x_{3}\right] d x_{3} .
\end{aligned}
$$


The integral in (B.5) can be solved by using [14, Eq. (8.350.2)], which reaches (15).

\section{Appendix C: Proof of Lemma 2}

We aim at deriving the asymptotic expressions for the two integrals in (A.1). For $\mathcal{I}_{1}$, we can rewrite (A.2) as

$$
\mathcal{I}_{1} \triangleq \int_{\epsilon}^{\infty} F_{X_{2 k}}\left(\frac{\gamma x_{4}}{\epsilon \bar{\gamma}}\right) f_{X_{4 k}}\left(x_{4}\right) d x_{4} .
$$

Then pulling (10), (17), and (C.1) together, we can get

$$
\mathcal{I}_{1}=\stackrel{\bar{\gamma}}{\approx}{ }^{\infty} \mathcal{B}_{1}\left(\frac{\gamma}{\bar{\gamma}}\right)^{m_{\mathrm{rd}}}
$$

where (C.2) immediately follows from [14, Eq. (8.350.2)]. For $\mathcal{I}_{2}$, by combining (17) and (A.5), it is straightforward to see that

$$
\mathcal{I}_{2} \stackrel{\bar{\gamma} \rightarrow \infty}{\approx} \mathcal{B}_{2}\left(\frac{\gamma}{\bar{\gamma}}\right)^{m_{\mathrm{rd}}}
$$

By adding (C.2) and (C.3) together, we can obtain (18).

\section{APPENDiX D: Proof of THEOREM 4}

From (B.2), we can obtain the following approximation after neglecting the small terms

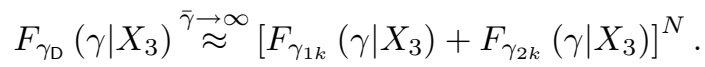

Combining Lemmas 1 and 2, we differentiate between two cases as follows:

- For $X_{3}<\epsilon$ : In this case, $F_{\gamma_{1 k}}\left(\gamma \mid X_{3}\right)$ is independent of $X_{3}$ as shown in (12), which yields

$$
F_{\gamma_{\mathrm{D}}}\left(\gamma \mid X_{3}\right) \stackrel{\bar{\gamma} \rightarrow \infty}{\approx} \mathcal{C}_{1}^{N}\left(\frac{\gamma}{\bar{\gamma}}\right)^{N \min \left(m_{\mathrm{sr}}, m_{\mathrm{rd}}\right)}
$$

where

$$
\mathcal{C}_{1}= \begin{cases}\mathcal{A}_{1}, & \text { for } m_{\mathrm{sr}}<m_{\mathrm{rd}} \\ \mathcal{B}, & \text { for } m_{\mathrm{sr}}>m_{\mathrm{rd}} \\ \mathcal{A}_{1}+\mathcal{B}, & \text { for } m_{\mathrm{sr}}=m_{\mathrm{rd}}\end{cases}
$$

- For $X_{3} \geq \epsilon$ : In this case, $F_{\gamma_{1 k}}\left(\gamma \mid X_{3}\right)$ contains $X_{3}$ as shown in (12), which yields

$$
F_{\gamma_{\mathrm{D}}}\left(\gamma \mid X_{3}\right) \stackrel{\bar{\gamma} \rightarrow \infty}{\approx} \mathcal{C}_{2}^{N}\left(\frac{\gamma}{\bar{\gamma}}\right)^{N \min \left(m_{\mathrm{sr}}, m_{\mathrm{rd}}\right)}
$$

where

$$
\mathcal{C}_{2}= \begin{cases}\mathcal{A}_{2} X_{3}^{m_{\mathrm{sr}}}, & \text { for } m_{\mathrm{sr}}<m_{\mathrm{rd}} \\ \mathcal{B}, & \text { for } m_{\mathrm{sr}}>m_{\mathrm{rd}} \\ \mathcal{A}_{2} X_{3}^{m_{\mathrm{sr}}}+\mathcal{B}, & \text { for } m_{\mathrm{sr}}=m_{\mathrm{rd}}\end{cases}
$$

By pulling (D.2) and (D.4) together with the PDF of $X_{3}$ given in (10), $\mathcal{J}_{1}$ and $\mathcal{J}_{2}$ in (B.1) can be approximated as, respectively

$$
\mathcal{J}_{1} \stackrel{\bar{\gamma} \rightarrow \infty}{\approx} F_{X_{3}}(\epsilon) \mathcal{C}_{1}^{N}\left(\frac{\gamma}{\bar{\gamma}}\right)^{N \min \left(m_{\mathrm{sr}}, m_{\mathrm{rd}}\right)}
$$

and

$$
\begin{aligned}
& \mathcal{J}_{2} \stackrel{\bar{\gamma}}{\approx} \approx\left(\frac{\gamma}{\bar{\gamma}}\right)^{N \min \left(m_{\mathrm{sr}}, m_{\mathrm{rd}}\right)} \\
& \times \begin{cases}\mathcal{A}_{2}^{N} \sum_{L-1, u, l, m_{\mathrm{sq}}, \lambda_{\mathrm{sq}}} \frac{L \lambda_{\mathrm{sq}}^{m_{\mathrm{sq}}}}{\Gamma\left(m_{\mathrm{sq}}\right)} & \\
\times \frac{\Gamma\left(m_{\mathrm{sr}} N+m_{\mathrm{sq}}+\vec{l}, \epsilon(u+1) \lambda_{\mathrm{sq}}\right)}{\left[(u+1) \lambda_{\mathrm{sq}}\right]_{\mathrm{sr}}^{m_{\mathrm{s}}+m_{\mathrm{sq}}+l}}, & \text { for } m_{\mathrm{sr}}<m_{\mathrm{rd}} \\
\mathcal{B}^{N}\left(1-F_{X_{3}}(\epsilon)\right), & \text { for } m_{\mathrm{sr}}>m_{\mathrm{rd}} \\
\sum_{k=0}^{N} \frac{\left(\begin{array}{l}
N \\
k
\end{array}\right) \mathcal{A}_{2}^{k} \mathcal{B}^{N-k}}{\sum_{\mathrm{siq}} \frac{L \lambda_{\mathrm{sq}}^{m_{\mathrm{sq}}}}{\Gamma\left(m_{\mathrm{sq}}\right)}} & \\
\times \frac{L-1, u, l, m_{\mathrm{sq}}, \lambda_{\mathrm{sq}}}{\Gamma\left(m_{\mathrm{sr}} k+m_{\mathrm{sq}}+\widetilde{l}, \epsilon(u+1) \lambda_{\mathrm{sq}}\right)} & \\
& \text { for } m_{\mathrm{sr}}=m_{\mathrm{rd}}\end{cases}
\end{aligned}
$$

Finally, the asymptotic CDF can be represented as (4), which concludes the proof.

\section{REFERENCES}

[1] A. Bletsas, A. Khisti, D. P. Reed, and A. Lippman, "A simple cooperative diversity method based on network path selection," IEEE J. Sel. Areas Commun., vol. 24, no. 3, pp. 659-672, 2006.

[2] A. Bletsas, H. Shin, and M. Z. Win, "Cooperative communications with outage-optimal opportunistic relaying," IEEE Trans. Wireless Commun., vol. 6, no. 9, pp. 3450-3460, Sep. 2007.

[3] E. Beres and R. S. Adve, "Selection cooperation in multisource cooperative networks," IEEE Trans. Wireless Commun., vol. 7, no. 1, pp. 118-127, 2008.

[4] S. S. Ikki and M. H. Ahmed, "Performance analysis of cooperative diversity wireless networks over Nakagami- $m$ fading channel," IEEE Commun. Lett., vol. 11, no. 4, pp. 334-336, 2007.

[5] A. Bletsas, H. Shin, and M. Z. Win, "Outage optimality of opportunistic amplify-and-forward relaying," IEEE Commun. Lett., vol. 11, no. 3, pp. 261-263, 2007.

[6] A. Bletsas, H. Shin, M. Z. Win, and A. Lippman, "Cooperative diversity with opportunistic relaying," in Proc. IEEE WCNC, Las Vegas, NV, Apr. 2006, pp. 1034-1039.

[7] J. Mitola et al., "Cognitive radio: making software radio more personal," IEEE Pers. Commun., vol. 6, no. 4, pp. 13-18, Aug. 1999.

[8] A. Ghasemi and E. S. Sousa, "Fundamental limits of spectrum-sharing in fading environments," IEEE Trans. Wireless Commun., vol. 6, no. 2, pp. 649-658, Feb. 2007.

[9] V. N. Q. Bao, T. Q. Duong, D. B. da Costa, G. C. Alexandropoulos, and A. Nallanathan, "Cognitive amplify-and-forward relaying with best relay selection in non-identical Rayleigh fading," IEEE Commun. Lett., 2013, in press.

[10] H. Ding et al., "Asymptotic analysis of cooperative diversity systems with relay selection in a spectrum sharing scenario," IEEE Trans. Veh. Technol., vol. 60, no. 2, pp. 457-472, Feb. 2011.

[11] K. J. Kim, T. Q. Duong, and X.-N. Tran, "Performance analysis of cognitive spectrum-sharing single-carrier systems with relay selection," IEEE Trans. Signal Process., vol. 60, no. 12, pp. 6435-6449, Dec. 2012.

[12] H. Tran, T. Q. Duong, and H.-J. Zepernick, "Performance analysis of cognitive relay networks under power constraint of multiple primary users," in Proc. IEEE GLOBECOM, Houston, TX, Dec. 2011, pp. 1-6.

[13] T. Q. Duong, P. L. Yeoh, V. N. Q. Bao, M. Elkashlan, and N. Yang, "Cognitive relay networks with multiple primary transceivers under spectrum-sharing," IEEE Signal Process. Lett., vol. 19, no. 11, pp. 741744, Nov. 2012

[14] I. S. Gradshteyn and I. M. Ryzhik, Table of Integrals, Series, and Products, 6th ed. San Diego, CA: Academic, 2000.

[15] Z. Chen, Z. Chi, Y. Li, and B. Vucetic, "Error performance of maximalratio combining with transmit antenna selection in flat Nakagami- $m$ fading channels," IEEE Trans. Wireless Commun., vol. 8, pp. 424-431, Jan. 2009. 